Int. J. Morphol.,

33(3):1032-1037, 2015

\title{
Irrigación de la Glándula Tiroides en una Muestra de Población Colombiana
}

\author{
Thyroid Gland Irrigation in a Sample of Colombian Population
}

\author{
Yobany Quijano Blanco* \& Ricardo Miguel Luque Bernal ${ }^{* * * * * *}$
}

QUIJANO, B. Y. \& LUQUE, B. R. M. Irrigación de la glándula tiroides en una muestra de población colombiana. Int. J. Morphol., 33(3):1032-1037, 2015.

RESUMEN: La glándula tiroides es un sitio frecuente de patologías, que requiere abordaje quirúrgico. Dichas técnicas, aunque bien establecidas, generan ocasionalmente complicaciones que, por lo general, se presentan por inexperiencia del cirujano o por variaciones anatómicas no previstas. El conocimiento y el estudio de estas variaciones son importantes, con el fin de limitar las complicaciones asociadas a procedimientos quirúrgicos de la glándula tiroides, disminuir la morbimortalidad asociada y mejorar la interpretación de las técnicas de imágenes diagnósticas. Se practicó un estudio descriptivo, con 67 bloques cervicocardiopulmonares y digestivos, en cadáveres de 64 hombres y 3 mujeres. Se realizó disección de la región cervical, a nivel de la glándula tiroides, identificando el origen, el trayecto y las relaciones de las arterias que irrigan a la glándula tiroides y, de igual manera, se buscó la presencia o no de la arteria tiroidea media (IMA). El total de 67 bloques estudiados evidenció que todos presentaban ambas arterias tiroideas bilateralmente. En todos los bloques analizados, la arteria tiroidea superior, se originó de la arteria carótida externa y siguió el recorrido clásico; la arteria tiroidea inferior, se inició en los 67 bloques del tronco tirocervical y, en 64 pacientes, el nervio laríngeo recurrente, se ubicó posterior a la arteria; solamente en 3 casos, se halló anteriormente. Se confirmó presencia de arteria tiroidea IMA en 4 pacientes, que corresponde al 6\% de los bloques. Los hallazgos de este estudio mantienen una gran correlación con la descripción clásica de la irrigación de la glándula tiroides en poblaciones occidentales. Con un origen y trayecto de ambas arterias tiroideas, que guardan una adecuada correlación con lo descrito previamente. La presencia de arteria tiroidea IMA fue inferior a lo reportado en la mayoría de las series de estudios.

PALABRAS CLAVE: Anatomía; Origen; Arterias tiroideas; Recurrente.

\section{INTRODUCCIÓN}

La tiroides es una glándula endocrina ubicada en el triángulo cervical anterior, fundamental en la homeostasis corporal, puesto que participa, de manera activa, en la regulación energético-metabólica y en el metabolismo del calcio, a través de la secreción de la hormona tiroidea (T3 y T4) y de la calcitonina (Latarjet \& Ruiz Liard, 2005).

Las patologías de la glándula tiroides tienen una alta prevalencia afectando, aproximadamente, a un 13,1\% de la población general (García Jiménez et al., 2007; Fardella et al., 2001), de las cuales, una tercera parte requiere manejo quirúrgico, ya sea con fines diagnósticos o terapéuticos; por tanto, las tiroidectomías, ya sea ismectomía parciales o totales, son procedimientos indispensables, para garantizar un adecuado estado de salud, de la población general (Pallardo, 2010).
A pesar de ser procedimientos milenarios, que se han perfeccionado con el tiempo y que la mortalidad es prácticamente nula en algunas series, aún las complicaciones quirúrgicas de tiroides sigue siendo una trampa para los cirujanos. Las causas más frecuentes de iatrogenia son la inexperiencia del cirujano y la no realización de una técnica cuidadosa, como lo refieren (Peralta et al., 1999; Sancho Fornos et al., 2001); sin embargo, la presencia de variaciones anatómicas desconocidas para los cirujanos es una causa tácita, de aparición de dichas complicaciones.

Entre las complicaciones más frecuentes producidas por cirugías de la glándula tiroides, se hallan hemorragias, infección, lesión de los nervios laríngeos y la resección accidental de las glándulas paratiroides, con la subsecuente hipocalcemia (Pallardo; Peralta et al.). En Colombia (Zúñiga

\footnotetext{
* Profesor titular. Facultad de Medicina. Universidad de Ciencias Aplicadas y Ambientales, Bogotá, Colombia.

** Instructor. Facultad de Medicina. Universidad de Ciencias Aplicadas y Ambientales, Bogotá, Colombia.

**** Profesor Asistente, Facultad de Medicina y Escuela de Ciencias de la Salud, Universidad del Rosario, Bogotá, Colombia.
} 
\& Sanabria, 2007), publicaron un estudio de seguimiento a pacientes con carcinoma papilar de tiroides tratados con tiroidectomía y detallan las complicaciones quirúrgicas, como lesión del nervio laríngeo recurrente presente entre el 0 y el $14 \%$ de las cirugías, la hipocalcemia secundaria a paratiroidectomia iatrogénica, entre el 0,4 y el 33\% de las tiroidectomías totales y el hematoma posoperatorio, con el 0,4 a 7,7\%. Estos episodios de iatrogenia, se han asociado, tradicionalmente, a falta de conocimiento de las variaciones anatómicas y, en el caso colombiano, tienen una frecuencia superior a la media internacional (Zúñiga \& Sanabria).

Los estudios, a nivel mundial, sobre la irrigación de la glándula tiroides y sus variaciones, han sido diversos y abundantes, comenzando con la descripción clásica, realizada por Neubauer en 1786, continuando con numerosos artículos, en diversas regiones del mundo. En estas investigaciones, se han observado un amplio rango de variaciones anatómicas, en lo referente a la irrigación de la glándula, tanto en el origen de las arterias como en su trayecto. Habitualmente, la glándula tiroides esta irrigada por la arteria tiroidea superior, siendo la primera ramificación de la arteria carótida externa e irriga, principalmente, la parte superior de la glándula y por la arteria tiroidea inferior, que es la rama principal del tronco tirocervical, que se deriva de la arteria subclavia. Adicionalmente, se ha reportado la presencia de una arteria inconstante, denominada la arteria tiroidea IMA, la cual, tiene una presencia en los diferentes reportes analizados, que varía entre un 10 y $20 \%$ de la población (Rojas \& Ballesteros, 2009; Latarjet \& Ruiz Liard; Gray et al., 1995; Ellis, 1997).

En Latinoamérica, se registra insuficiente literatura, que permita dar cuenta de las variaciones anatómicas de las estructuras vasculares que irrigan la glándula tiroides. Dada la composición étnica de la región, que la hace diferente al resto del mundo y, por tanto, genera cambios en la frecuencia de variaciones anatómicas y en los tipos de variaciones, que se han reportado a nivel mundial. Es necesario conocer y exponer las variaciones que están presentes en esta región, particularmente en Colombia, donde las complicaciones de las cirugías de tiroides presentan una frecuencia mayor a la media mundial. Por lo anterior, esta investigación muestra las características de la irrigación arterial de la glándula tiroides en una muestra de población colombiana.

\section{MATERIAL Y MÉTODO}

Se realizó un estudio descriptivo, usando 67 bloques cervicocardiopulmonares y digestivos de población colombiana; 64 bloques pertenecían a cadáveres de género mas- culino y 3 , femenino, seleccionados por muestreo por conveniencia. Como criterios de exclusión del estudio, se tuvieron traumas cervicales, bloques con malformaciones congénitas de la glándula tiroides o de las estructuras adyacentes, que fuesen evidentes a la inspección o que presentaran lesiones tumorales, a dicho nivel.

La disección, se efectuó usando la técnica sugerida por Loukas et al. (2005). Se expone la glándula tiroides, retirando los músculos infrahioideos; luego, se procedió a disecar la arteria carótida común, identificando sus ramas externa e interna. Posteriormente, se procedió a seguir el trayecto de la arteria tiroidea superior, desde su origen hasta la glándula; en seguida, desde la glándula, se procedió a disecar la arteria tiroidea inferior hasta su origen y se diseca simultáneamente el nervio laríngeo recurrente, identificando la relación de éste con la arteria. A continuación, se buscó la presencia de arteria tiroidea media, a partir del istmo, hasta la identificación de su origen y relaciones, en los casos en que estuviese presente.

El trabajo siguió todas las normas éticas contenidas en la declaración de Helsinki (Asociación Médica Mundial, 2008) para investigación biomédica, dando un trato respetuoso a los cadáveres utilizados. Se garantizó la confidencialidad de los datos, y la integridad y la dignidad de las personas fallecidas. Se realizó previa aprobación del comité de bioética institucional y se procedió al cumplimiento estricto de todos los aspectos normativos, contenidos en el decreto 786 de 1990 y en la resolución 8430 de 1993, del Ministerio de Salud Nacional.

\section{RESULTADOS}

Al realizar la disección de la glándula tiroides, se evidenció que la arteria tiroidea superior siempre tuvo su origen en la arteria carótida externa. En algunos bloques, se observó que, posterior a su origen, podían dar una o dos ramas; sin embargo, no se advirtió la presencia de tronco tirolingual ni tirofacial, en este estudio (Fig. 1).

La arteria tiroidea superior, se originó, en todos los casos, de la cara medial de la arteria carótida externa y siguió un trayecto medial e inferior, hasta abordar la glándula tiroides, por la cara lateral y superior de sus lóbulos; en dos plastrones, se evidenció la emergencia de la arteria laríngea superior de la cara superior de la arteria tiroidea superior (Fig. 2)

Al disecar la arteria tiroidea inferior, se percibió que, en todos los casos, se originaban en el tronco tirocervical y 
se dirigían medial y discretamente superior, para llegar a la glándula tiroides, a nivel del polo inferior de sus lóbulos (Fig. 3). En 5 pacientes, 7\%, el nervio laríngeo recurrente, se ubicó anterior a la arteria tiroidea inferior; entre las ramas de la arteria, en 6 pacientes, $8 \%$ y, en el restante, $85 \%$, se presentó la disposición clásica, donde el nervio se halla posterior a la arteria.

La arteria tiroidea IMA estuvo presente en 4 bloques 6\% (Bloques 13, 17, 20 y 63), proviniendo de un bloque directamente del cayado de la aorta (Bloque 13), mientras en los 3 restantes, se originaron del tronco braquiocefálico arterial. Dicha arteria ascendió, en todos los casos, por la cara anterior de la tráquea, abordando la glándula tiroides, a nivel de la cara inferior del istmo (Fig. 4).

$\mathrm{Al}$ observar la glándula tiroides, se encontró que en 5 de ellas se presentaban un lóbulo piramidal $(7,5 \%)$ (Bloques $11,18,20,35$ y 63) y, una, presentaba agenesia del istmo (1,5\%) (Fig. 5, Tabla I).

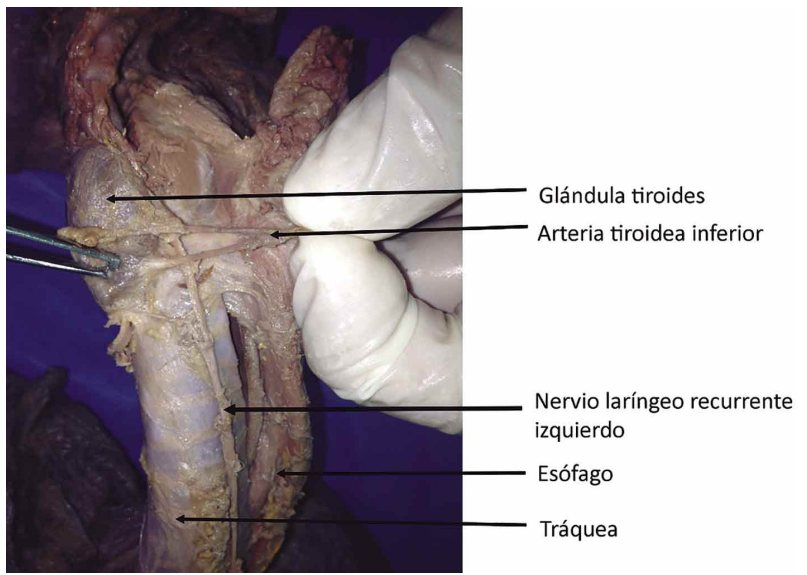

Fig. 1. Emergencia de la arteria tiroidea superior desde la carótida externa, dando dos ramas y su relación con el nervio laríngeo recurrente.

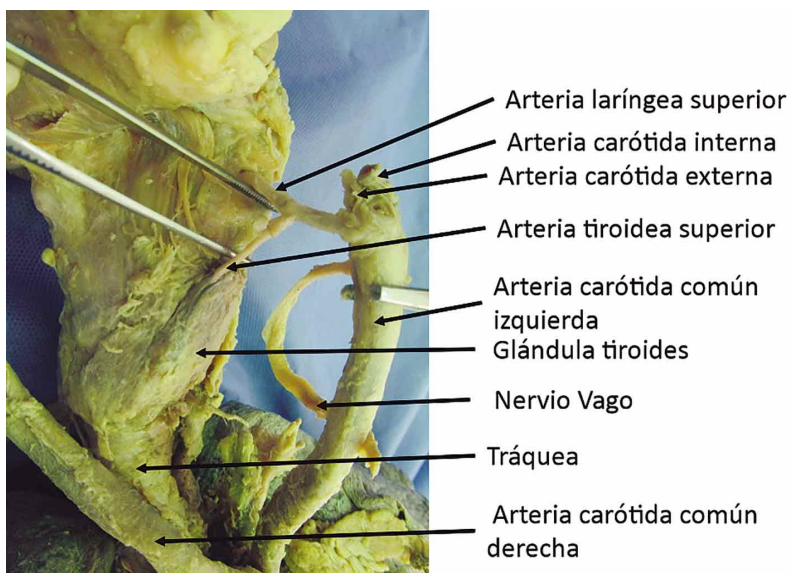

Fig. 2. Emergencia de la arteria tiroidea superior desde la carótida externa, dando dos ramas. Arriba la arteria laríngea superior e, inmediatamente inferior, la arteria tiroidea superior.

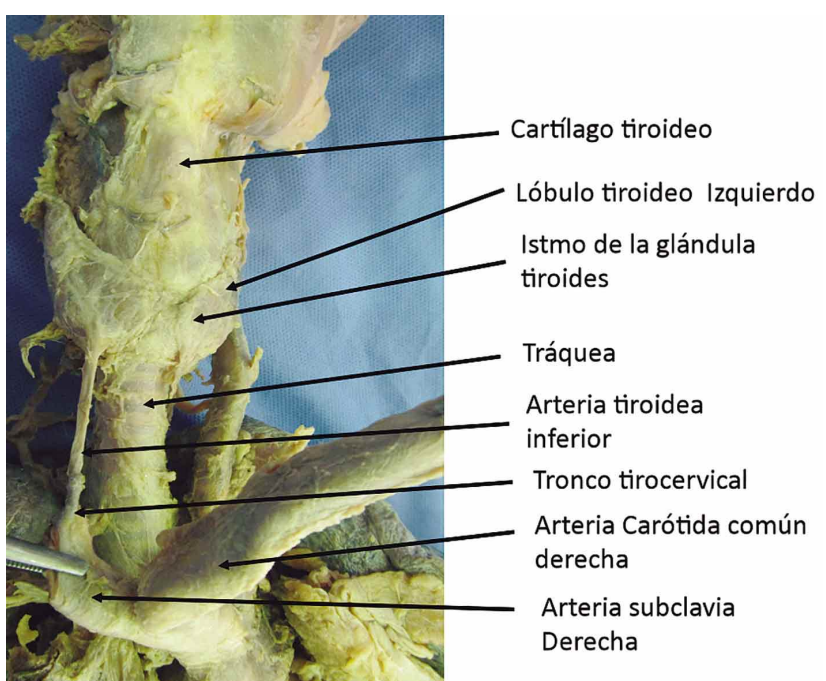

Fig. 3. Emergencia de la arteria tiroidea inferior desde la arteria subclavia a través del tronco tirocervical.

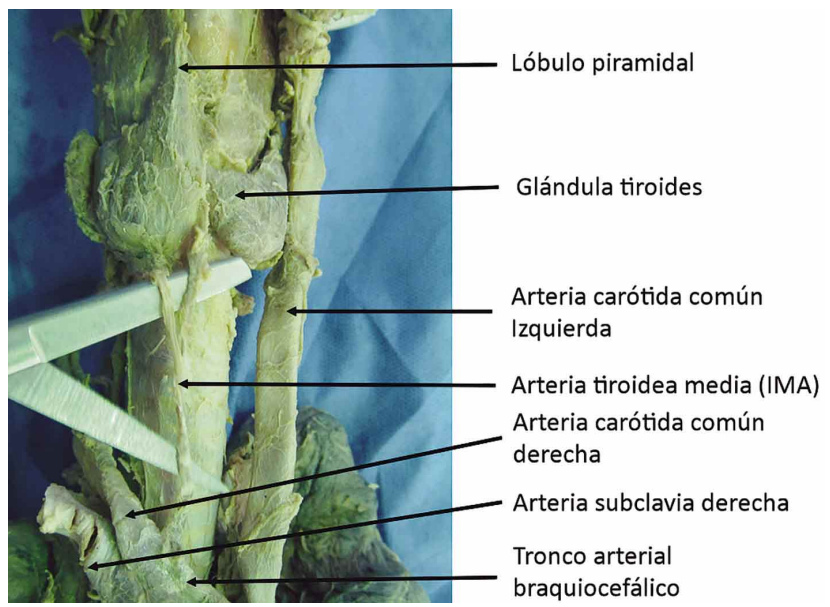

Fig. 4. Emergencia de la arteria tiroidea media (IMA) desde el tronco arterial braquiocefálico.

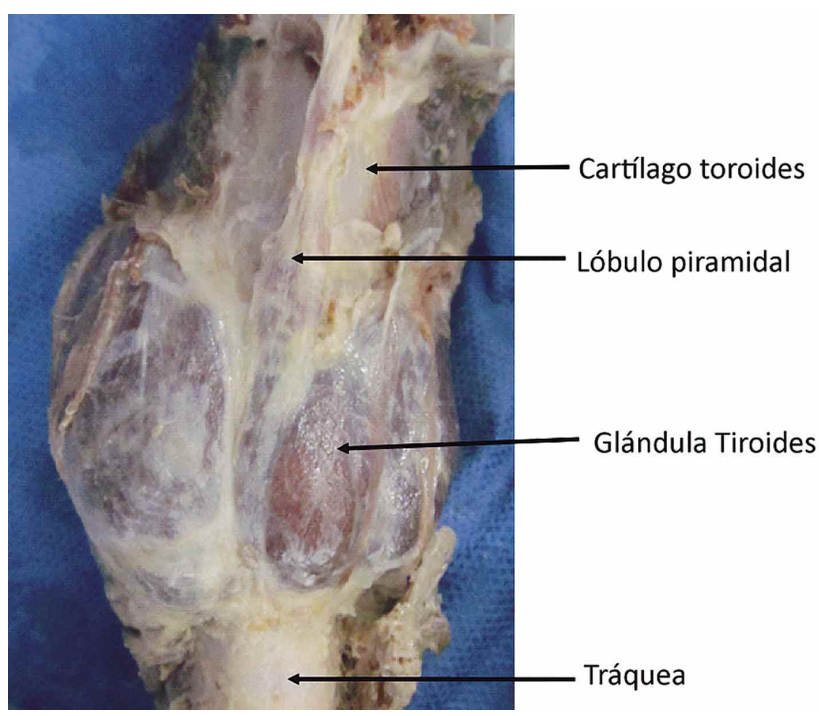

Fig. 5. Presencia del lóbulo piramidal tiroideo. 
Tabla I. Origen de las arterias tiroideas.

\begin{tabular}{|c|c|c|c|c|c|}
\hline Arteria & $\begin{array}{l}\text { Número de } \\
\text { casos }\end{array}$ & Origen & Porcentaje & Variaciones & Porcentaje \\
\hline \multirow[t]{2}{*}{ Tiroidea superior } & 67 & Arteria carótida externa & $100 \%$ & 1 caso 2 ramas & $1,5 \%$ \\
\hline & & & & 1 caso 1 rama & $1,5 \%$ \\
\hline \multirow[t]{2}{*}{ Tiroidea inferior } & 67 & Tronco tirocervical & $100 \%$ & 2 casos 2 ramas & $3 \%$ \\
\hline & & & & 4 casos 1 rama & $7,5 \%$ \\
\hline Tiroidea media IMA & 4 & --- & $6 \%$ & No & No \\
\hline Tiroidea IMA & 3 & Tronco Arterial Braquiocefálico & $75 \%$ & No & No \\
\hline Tiroidea IMA & 1 & Cayado de la a orta & $25 \%$ & No & No \\
\hline
\end{tabular}

$\mathrm{CE}=\mathrm{A}$. carótida externa, $\mathrm{CC}=\mathrm{A}$. carótida común, $\mathrm{BC}=$ Bifurcación carotidea, $\mathrm{TTC}=$ Tronco tirocervical,

$\mathrm{TAB}=$ Tronco arterial braquiocefálico, $\mathrm{SUB}=$ Arteria subclavia. $\mathrm{A} . \mathrm{A}=$ Arco de la arteria aorta.

\section{DISCUSIÓN}

La irrigación de la glándula tiroides es variada, amplia y sujeta a múltiples variaciones anatómicas, tanto en el número de arterias encargadas de la irrigación como en origen de estas, su trayecto y relaciones anatómicas. Entre los factores que explicarían dichas variaciones, diversos autores las han relacionado con el grupo étnico de las personas (Toni et al., 2003, 2004, 2005); sin embargo, en las descripciones de la variable "grupo étnico" no utilizan criterios claros, que la definan más allá de la procedencia del individuo.

En este trabajo, el origen de la arteria tiroidea superior, en todos los bloques estudiados, se puede clasificar como tipo III, de acuerdo a la clasificación propuesta por Vázquez et al. (2009), dado que la arteria tiroidea superior siempre se originó de la arteria carótida externa. En contraste con lo encontrado por Vázquez et al., quienes describieron que en el $49 \%$ de los casos la arteria tiroidea superior se originaba de la bifurcación de la carótida (tipo I), lo hace $27 \%$ de la carótida común (tipo II), directamente de la carótida externa, solamente se originó el $23 \%$ y lo clasificaron como tipo III. Un metaanálisis realizado por Toni et al. (2004), evidenciaron asimetría en el origen de las arterias tiroideas superiores, ya que al lado derecho, su origen era predominantemente en la arteria carótida externa, mientras que, en el izquierdo, de la arteria carótida común. Por el contrario, en este estudio, no se evidenció asimetría en el origen de las arterias tiroideas superiores derecha e izquierda.

La descripción clásica de la arteria tiroidea inferior refiere su génesis, ya sea en la arteria subclavia o en el tronco tirocervical, de acuerdo al origen étnico de la población estudiada (Toni et al., 2005). Así, en poblaciones asiáticas es más frecuente el origen en el tronco tirocervical, mientras en caucásica es más habitual en la arteria subclavia. De la misma forma, en población asiática es frecuente que la arteria tiroidea inferior esté ausente, en contraste con la población caucásica, donde, por lo general, se encuentra presente (Toni et al., 2004). En el presente estudio, se evidenció que todos los bloques tenían arteria tiroidea inferior y siempre se origina en el tronco tirocervical, lo que implica que, al menos en la muestra tomada en este estudio, el origen y la distribución de la arteria tiroidea inferior tendría un comportamiento diferente, a lo descrito en los otros grupos étnicos. Respecto a la relación de la arteria tiroidea inferior con el nervio laríngeo recurrente, primó la ubicación posterior del nervio respecto a la arteria, presente en el $85 \%$ de las arterias estudiadas. Contrario a lo reportado en el estudio de Yalcin (2006), donde el nervio, al lado izquierdo, estaba posterior, en el 52\% de los casos; anterior, en el 13\% y, pasaba entre sus ramas, en el $26 \%$, mientras al lado derecho, se encontraba posterior, en el $23 \%$ de los casos; anterior, en el $25 \%$ y, entre las ramas, en el 36\%. En otro estudio (Carter et al., 2012), reportaron que en el $91 \%$ de los caucásicos, el nervio laríngeo recurrente se ubica inferior a la arteria tiroidea inferior; $4 \%$, anterior y, un 5\%, entre sus ramas, no haciendo distinción por lateralidad. Dado que en este estudio se analizaron diversos grupos étnicos, se evidenció una distribución similar en casi todos, con excepción de los afro-caribeños y de los negros-africanos, en quienes el porcentaje de arteria tiroidea inferior anterior al nervio era sólo de $57 \%$ y un $74 \%$, respectivamente.

La arteria tiroidea IMA es inconstante y se ha reportado con una frecuencia variable, de acuerdo a los diversos estudios. El presente estudio halló una frecuencia de la arteria tiroidea IMA del 6\%, donde el 75\% emergió del tronco braquiocefálico y, del cayado de la aorta directamente, el $25 \%$. En contraste con el estudio realizado por Kitagawa (1993), quien observó esta arteria, en el 16\% de los casos, registró que el $80 \%$ surgía del tronco braquiocefálico y, el $20 \%$ restante, de la arteria carótida común (Tabla II). 
Tabla II. Irrigación de la glándula tiroides por diversos autores.

\begin{tabular}{|c|c|c|c|c|c|c|}
\hline Estudio & Procedencia & $\begin{array}{l}\text { Tipo de } \\
\text { estudio }\end{array}$ & $\begin{array}{c}\text { n de } \\
\text { tiroides } \\
\text { estudiadas }\end{array}$ & $\begin{array}{c}\text { Origen } \\
\text { ATS }\end{array}$ & Origen ATI & $\begin{array}{c}\text { Origen } \\
\text { ATM (IMA) }\end{array}$ \\
\hline Kitagawa (1993) & Japón & $\begin{array}{l}\text { Disección } \\
\text { fetos }\end{array}$ & 37 & $\begin{array}{l}\text { BC: } 26,5 \% \\
--- \\
\text { CC: } 11,7 \% \\
\text { CE: } 61,8 \%\end{array}$ & $\begin{array}{l}\text { TTC: } 93,6 \% \\
\text { Ausente: } 6,4 \% \\
--\end{array}$ & $\begin{array}{l}\text { TBC: } 12 \% \\
\text { C.C: } 3 \% \\
--\end{array}$ \\
\hline Toni et al. (2003) & Italia & $\begin{array}{l}\text { Disección } \\
\text { cadáveres }\end{array}$ & 123 & CE: $100 \%$ & $\begin{array}{l}\text { TTC: } 94,8 \% \\
\text { SUB: } 5,2 \%\end{array}$ & --- \\
\hline Vázquez et al. (2005) & Reino Unido & $\begin{array}{l}\text { Disección } \\
\text { cadáveres } \\
\text { descriptivo }\end{array}$ & 165 & $\begin{array}{l}\text { BC: } 49,3 \% \\
\text { CC: } 26,6 \% \\
\text { CE: } 23,2 \%\end{array}$ & NA & NA \\
\hline Toni et al. (2004) & Caucasoide & Metaanálisis & 763 & $\begin{array}{l}\text { CE: } 69 \% \\
\text { CC: } 31 \%\end{array}$ & --- & --- \\
\hline & Asiáticos & & 311 & CE: $58 \%$ & & \\
\hline Toni et al. (2005) & Caucasoide & $\begin{array}{l}\text { Metaanálisis } \\
\text { Disección }\end{array}$ & $\begin{array}{c}6285 \\
847\end{array}$ & & $\begin{array}{l}\text { TTC: } 90 \% \\
\text { SUB: } 10 \%\end{array}$ & --- \\
\hline Natsis et al. (2011) & $\begin{array}{l}\text { Asiáticos } \\
\text { Grecia }\end{array}$ & $\begin{array}{l}\text { cadáveres } \\
\text { Disección } \\
\text { cadáveres }\end{array}$ & 100 & $\begin{array}{l}\text { BC: } 49 \% \\
\text { CC: } 12 \% \\
\text { CE: } 39 \%\end{array}$ & $\begin{array}{l}\text { TTC: } 95 \% \\
\text { NA }\end{array}$ & NA \\
\hline Hayashi et al. (2005) & Japón & $\begin{array}{l}\text { Disección } \\
\text { cadáveres }\end{array}$ & 98 & $\begin{array}{l}\text { CC: } 30 \% \\
\text { CE: } 69 \% \\
\text { TTC: } 1 \%\end{array}$ & NA & NA \\
\hline Shintani et al. (1999) & Japón & $\begin{array}{l}\text { Disección } \\
\text { cadáveres }\end{array}$ & 58 & $\begin{array}{l}\text { CC: } 0 \% \\
\text { BC: } 0 \% \\
\text { CE: } 100 \%\end{array}$ & NA & NA \\
\hline $\begin{array}{l}\text { Presente } \\
\text { estudio }\end{array}$ & Colombia & $\begin{array}{l}\text { Disección } \\
\text { cadáveres }\end{array}$ & 67 & CE: $100 \%$ & TTC: $100 \%$ & $\begin{array}{l}6 \%(4 \text { casos }) \\
\text { TAB: } 75 \%\end{array}$ \\
\hline
\end{tabular}

(3)

A.A: $25 \%(1)$

$\overline{\mathrm{CE}}=\mathrm{A}$. carótida externa, $\mathrm{CC}=\mathrm{A}$. carótida común, $\mathrm{BC}=$ Bifurcación carotidea, $\mathrm{TTC}=$ Tronco tirocervical, TAB: Tronco arterial braquiocefálico, $\mathrm{SUB}=$ Arteria subclavia. A.A= Arco de la arteria aorta. NA= No aplica.

En cuanto a la morfología de la glándula tiroides, se manifestó una descripción clásica en la mayoría de los cadáveres estudiados, $92,5 \%$, mientras en el 7,5\% restante, se observó la presencia de un lóbulo piramidal. Contrastando con el estudio realizado por Braun et al. (2007), quienes practicaron un estudio en 60 cadáveres, 28 mujeres y 32 hombres, percibiendo para ambos sexos, una frecuencia igual o superior al $50 \%$ de glándulas tiroides con lóbulos piramidales.

Las variaciones registradas en este estudio respecto a otras investigaciones, probablemente, se deban a los diferentes orígenes de los grupos poblacionales estudiados aquí, respecto a los otros, así como también por el tamaño y la selección de las muestras realizadas en los diversos estudios; no obstante, es claro que los resultados hallados en este estudio no se alejan de las descripciones clásicas aplicadas en población occidental, pero sí lo hacen de investigaciones de población de origen asiático.
En este estudio, se tomó como muestra 67 bloques, siendo uno de los pocos estudios que hay de este tipo en población latinoamericana, lo que permite iniciar comparaciones por grupos étnicos, dado que la población latinoamericana y, particularmente, la colombiana, tienen una composición étnica específica, que hacen difícil la extrapolación de datos tomados de otras poblaciones.

QUIJANO, B. Y. \& LUQUE, B. R. M. Thyroid gland irrigation in a sample of Colombian population. Int. J. Morphol., 33(3):1032-1037, 2015.

SUMMARY: The thyroid gland is a frequent site of pathology that requires surgical approach. Such techniques, although well-established is occasionally complicated, which usually occurs because of the inexperienced surgeon or unintended anatomical variations. The knowledge and study of these variations are important in order to limit the complications associated with surgical procedures of the thyroid gland, reduce associated morbidity and mortality and improve the performance of diagnostic imaging techniques. Adescriptive study was performed with 67 digestive and cervico cardiopulmonary blocks, in cadavers 64 men and 3 women. Dissection of 
the cervical region was performed at the level of the thyroid gland, identifying the origin, course and relations of the arteries supplying the thyroid gland and, likewise, sought the presence or absence of the middle thyroid artery (IMA). The total of 67 blocks studied showed that all had both thyroid arteries bilaterally. In all blocks analyzed, the superior thyroid artery originated from the external carotid artery and followed the classic route; the inferior thyroid artery, started in the 67 blocks thyrocervical trunk and in 64 patients, the recurrent laryngeal nerve was located posterior to the artery; only in 3 cases, it was found above. IMA presence of thyroid artery in 4 patients, corresponding to $6 \%$ of the blocks was confirmed. The findings of this study remain highly correlated with the classical description of the irrigation of the thyroid gland in Western populations, in an origin and course of both thyroid arteries that have a good correlation with those previously described. The presence of thyroid artery IMA was lower than that reported in most series of studies.

KEY WORDS: Anatomy; origin; Thyroid arteries; Recurrent.

\section{REFERENCIAS BIBLIOGRÁFICAS}

Asociación Médica Mundial. Declaración de Helsinki de la Asociación Médica Mundial. Principios éticos para las investigaciones médicas en seres humanos. Seúl, 59 $9^{\circ}$ Asamblea General, Asociación Médica Mundial, 2008. Disponible en: http://www.wma.net/es/30publications/ 10policies/b3/17c_es.pdf

Braun, E. M.; Windisch, G.; Wolf, G.; Hausleitner, L. \& Anderhuber, F. The pyramidal lobe: clinical anatomy and its importance in thyroid surgery. Surg. Radiol. Anat., 29(1):21-7, 2007.

Carter, C. P.; Collier, J. \& Cheng, L. The anatomical variation between recurrent laryngeal nerve and inferior thyroid artery among different racial groups who underwent thyroid and parathyroid surgery. $B r . J$. Oral Maxillofac. Surg., 50(Suppl. 1):S51, 2012.

Ellis, H. Clinical Anatomy. 3rd edn. J. Anat., 190(Pt. 4):631-2, 1997.

Fardella B. C.; Poggi, M. H.; Gloger, K. S.; Rojas, O. A.; Velásquez, A. C. G.; Barroileth, D. S.; Figueroa, R. R.; Alvarez, B. C.; Salgado, M. C.; Gajardo, J. C.; Foradori, C. A. \& Montero, L. J. Alta prevalencia de enfermedad tiroidea subclínica en sujetos que concurren a control de salud. Rev. Méd. Chile, 129(2):155-60, 2001.

García Jiménez, E.; Ocaña Arenas, A. \& Torres Antiñolo, A. Guía de seguimiento farmacoterapéutico en hipotiroidismo e hipertiroidismo. Monografía sobre disfunción tiroidea. Granada, Grupo de Investigación en Atención Farmacéutica. Universidad de Granada, 2007. pp.1116.

Gray, H.; Williams, P. L. \& Bannister, L. H. Gray's anatomy: the anatomical basis of medicine and surgery. 38th ed. New York, Churchill Livingstone, 1995.

Hayashi, N.; Hori, E.; Ohtani, Y.; Ohtani, O.; Kuwayama, N. \& Endo, S. Surgical anatomy of the cervical carotid artery for carotid endarterectomy. Neurol. Med. Chir. (Tokyo), 45(1):25-9, 2005.

Kitagawa, W. Arterial supply of the thyroid gland in the human fetuses. Nihon Ika Daigaku Zasshi, 60(3):140-55, 1993.

Latarjet, M. \& Ruiz Liard, A. Anatomía Humana. Buenos Aires, Médica Panamericana, 2005.
Loukas, M.; Benninger, B. \& Tubbs, S. Gray Guía Fotográfica de Disección del Cuerpo Humano. Barcelona, Elsevier, 2005. pp.296-309.

Natsis, K.; Raikos, A.; Foundos, I.; Noussios, G.; Lazaridis, N. \& Njau, S. N. Superior thyroid artery origin in Caucasian Greeks: A new classification proposal and review of the literature. Clin. Anat., 24(6):699-705, 2011.

Pallardo, S. L. F. Endocrinología Clínica. $2^{\text {a }}$ ed. Madrid, Díaz de Santos, 2010. pp.39-44.

Peralta, P. R.; Fleites, G. G.; Cassola, S. J. R.; Guerra, M. J. L. \& Collado, O. J. C. Servicio de Cirugía Esplácnica. Instituto Nacional de Oncología y Radiobiología. Cirugía tiroidea: principios anatómicos y técnicos para reducir complicaciones. Rev. Cuba. Oncol., 15(2):81-8, 1999.

Rojas, O. J. D. \& Ballesteros, A. L. E. Branches arise of the aortic arch in human fetus. a descriptive direct study in colombian population. Int. J. Morphol., 27(4):989-96, 2009.

Sancho Fornos, S.; Vaqué Urbaneja, J.; Ponce Marco, J. L.; Palasí Giménez, R. \& Herrera Vela, C. Complicaciones de la cirugía tiroidea. Cir. Esp., 69(3):198-203, 2001.

Shintani, S.; Terakado, N.; Alcalde, R. E.; Tomizawa, K.; Nakayama, S.; Ueyama, Y.; Ichikawa, H.; Sugimoto, T. \& Matsumura, T. An anatomical study of the arteries for intraarterial chemotherapy of head and neck cancer. Int. J. Clin. Oncol., 4(6):327-30, 1999.

Toni, R.; Della Casa, C.; Mosca, S.; Malaguti, A.; Castorina, S. \& Roti, E. Anthropological variations in the anatomy of the human thyroid arteries. Thyroid, 13(2):183-92, 2003.

Toni, R.; Della Casa, C.; Castorina, S.; Malaguti, A.; Mosca, S.; Roti, E.; Valenti, G. A meta-analysis of superior thyroid artery variations in different human groups and their clinical implications. Ann. Anat., 186(3):255-62, 2004.

Toni, R.; Casa, C. D.; Castorina, S.; Roti, E.; Ceda, G. \& Valenti, G. A meta-analysis of inferior thyroid artery variations in different human ethnic groups and their clinical implications. Ann. Anat., 187(4):37185, 2005.

Vázquez, T.; Cobiella, R.; Maranillo, E.; Valderrama, F. J.; McHanwell, S.; Parkin, I. \& Sañudo, J. R. Anatomical variations of the superior thyroid and superior laryngeal arteries. Head Neck, 31(8):1078-85, 2009.

Yalcin, B. Anatomic configurations of the recurrent laryngeal nerve and inferior thyroid artery. Surgery, 139(2):181-7, 2006.

Zúñiga, S. \& Sanabria, Á. Complicaciones y recurrencia en el tratamiento del carcinoma papilar de tiroides. Experiencia del Instituto Nacional de Cancerología. Rev. Colomb. Cir., 22(3):166-74, 2007.

\section{Dirección para Correspondencia:}

Dr. Yobany Quijano Blanco

Facultad de Medicina

Universidad de Ciencias Aplicadas y Ambientales U.D.C.A

Bogotá

COLOMBIA

Recibido : 30-09-2014

Aceptado: 13-07-2015

Email: globdimorf@udca.edu.co 